\title{
AN EFFICIENT IMPLEMENTATION OF TRACKING USING KALMAN FILTER FOR UNDERWATER ROBOT APPLICATION
}

\author{
Nagamani Modalavalasa ${ }^{1}$, G SasiBhushana $\operatorname{Rao}^{2}$, K. Satya Prasad ${ }^{3}$ \\ ${ }^{1}$ Dept.of ECE, SBTET, Andhra Pradesh, INDIA \\ mani.modalavalasa@gmail.com \\ ${ }^{2}$ Dept. of ECE, Andhra University, Visakhapatnam, Andhra Pradesh, INDIA \\ ${ }^{3}$ Dept.of ECE, Jawaharlal Nehru Technological University Kakinada, Kakinada, INDIA
}

\begin{abstract}
The exploration of oceans and sea beds is being made increasingly possible through the development of Autonomous Underwater Vehicles (AUVs). This is an activity that concerns the marine community and it must confront the existence of notable challenges. However, an automatic detecting and tracking system is the first and foremost element for an AUV or an aqueous surveillance network. In this paper a method of Kalman filter was presented to solve the problems of objects track in sonar images. Region of object was extracted by threshold segment and morphology process, and the features of invariant moment and area were analysed. Results show that the method presented has the advantages of good robustness, high accuracy and real-time characteristic, and it is efficient in underwater target track based on sonar images and also suited for the purpose of Obstacle avoidance for the AUV to operate in the constrained underwater environment.
\end{abstract}

\section{KEYWORDS}

Autonomous Underwater Vehicle, Tracking, SONAR, Threshold, Obstacle avoidance

\section{INTRODUCTION}

Autonomous underwater vehicles (AUVs) have the potential to revolutionize our access to the oceans to address critical problems facing the marine community such as underwater search and mapping, climate change assessment, marine habitat monitoring, and shallow water mine countermeasures. Navigation is one of the primary challenges in AUV research today.

Navigation is an important requirement for any type of mobile robot, but this is especially true for autonomous underwater vehicles. Good navigation information is essential for safe operation and recovery of an AUV. For the data gathered by an AUV to be of value, the location from which the data has been acquired must be accurately known. Some of the important concerns for AUV navigation, such as the effects of acoustic propagation are unique to the ocean environment.

The goal of this paper is the Surveillance using Imaging Sonar Data for Underwater Robot Application based on Kalman filter. In this paper, the images from the Compressed High Intensity Radar Pulse (CHIRP) Sonar are used for the analysis. CHIRP Sonars are the active Sonars invented to overcome the limitations of conventional monotonic Sonars. In conventional Sonars when the separation of the targets is less than the range resolution, then it displays as a single 
large combined target rather than the multiple smaller targets. On the other hand, if we use the smaller transmission pulse to increase the range resolution, then the maximum range obtained decreases due to less energy. In order to overcome this problem, CHIRP Sonars have been developed and made use of. . In this paper Sector Scan SONAR images are taken as input data and processed for obtaining the tracking results.

Several methods are available for tracking the objects in the image sequences received from the Sonar fitted on the AUV. These methods are not suitable for undertaking the collision avoidance if the AUV is required to be controlled in the constrained underwater environment. The time required for extraction of target parameters and for subsequent tracking becomes an important criterion because the AUV is required to be maneuvered well before the collision occurs. A comparison of the commonly used algorithms for data association and tracking namely Nearest Neighbour Kalman filter (NNKF) and Probabilistic data association filter (PDAF) is made in ref. [1] for single target tracking in clutter. In this paper, tracking of the objects (detected in the sequence of images received from Sonar) based on their centroids has been presented. Accordingly the calculation of the centroid, tracking of the objects and the calculation of the trajectories has been presented.

\section{Image Processing Model Based On Centroid Of The ObJect}

In image-based air traffic control or air defense system, automatic detection and tracking of targets are extremely important for their safety or early warning. In such scenario, the sensor images are often cluttered, dim, spurious or noisy due to the fact that the distances to targets from the control centre are large. Tracking problems involve processing measurements from a target of interest and producing at each time step, an estimate of the target's current position and velocity vectors. Uncertainties in the target motion and in the measured values, usually modelled as additive random noise, lead to corresponding uncertainties in the target state. Also, there is additional uncertainty regarding the origin of the received data, which may or may not include measurements from the targets and may be due to random clutter (false alarms). This leads to the problem of data association [2]. In this situation tracking algorithms have to include information on detection and false alarm probabilities. This approach provides a method of centroid tracking and target identity estimation using image SONAR data.

The Centroid tracking combines both object and motion recognition characteristics for practical target tracking from imaging sensors. The characteristics of the image considered are the intensity and size of the cluster. The pixel intensity is discretised into several layers of gray level intensities and it is assumed that sufficient target pixel intensities are within the limits of certain target layers. The centroid tracking implementation involves the conversion of the image into a binary image and applying upper and lower threshold limits for the "target layers". The binary target image is then converted to clusters by using nearest neighbour criterion. If the target size is known, then it is used to set limits for removing those clusters that differ sufficiently from the size of the target cluster to reduce computational complexity. The centroid of the clusters is then calculated and this information is used for tracking the target. The Centroid tracking involves the following steps:

a. Pre-processing to remove the noise / blur from the images. (In present day applications this step is generally performed by the Sonar)

b. Identifying potential targets by image segmentation methods. Real Sonar image and the image after segmentation are shown in Figure 1 and Figure 2. In this, the image is segmented into objects, shadow and sea bottom reverberation regions and then the edges of the object are extracted [3].

c. Calculation of the centroids for all the detected objects. 
d. The steps (a) to (c) are performed on all subsequent images

e. Identification of the moving and stationary objects.

f. Determination of the association of the moving objects based on the maximum speed criterion.

g. Tracking of the moving objects using Kalman Filter $\quad[4,5]$

h. Calculation of the trajectory.

i. Calculation of Collision course by taking the own speed and direction into consideration [6].

j. $\quad$ Finally executing the manoeuvring commands to AUV

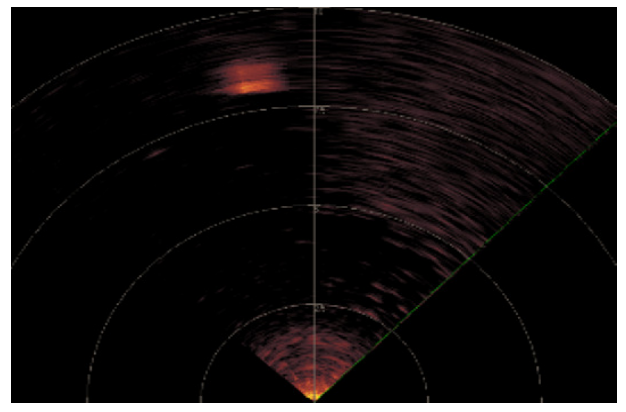

Figure 1. Real SONAR image

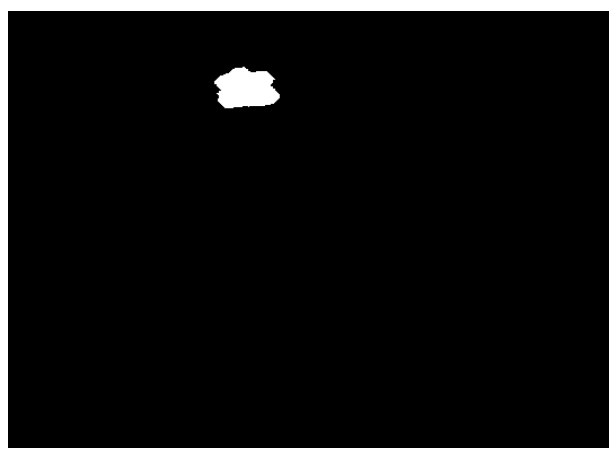

Figure 2. Object identification from the proposed segmentation method

\section{Tracking Algorithm}

The sequence of images can either be processed in real-time, coming directly from a video camera for example, or it can be performed on a recorded set of images. The implementation of this paper uses recorded image sequences although the theory can be applied to both types of applications. The target to be tracked might be a complete object or a small area on an object. In either case, the feature of interest is typically contained within a target region. In this paper will consider target centroid positions across the image plane. The position will be described in $\mathrm{X}-\mathrm{Y}$ coordinates in pixel units on the image (i.e. image coordinates). 
Once objects are detected, we extract their outline and track their centroids across the image plane using separate linear Kalman filters to estimate their $\mathrm{x}$ and $\mathrm{y}$ coordinates. The Kalman filter provides a general solution to the recursive minimised mean square linear estimation problem. The mean square error will be minimised as long as the target dynamics and the measurement noise are accurately modelled. In addition, the Kalman filter provides a convenient measure of the estimation accuracy through the covariance matrix, and the gain sequence automatically adapts to the variability of the data. Figure 3 explains the steps, those have been implemented to track the objects in the images received from the imaging Sonar of AUV. A linear, discrete-time dynamic system describing the target parameters estimation is shown in Figure 4.

\section{KALMAN FILTER}

Derivations of the Kalman filter are available in the literature, e.g. [7], [8] and [9]. The transition from one state to the next could be described in many ways. These different alternatives can be grouped into linear and non-linear functions describing the state transition. Although it is possible to handle either of these transition types, the standard Kalman filter employs a linear transition function. The extended Kalman filter (EKF) allows a non-linear

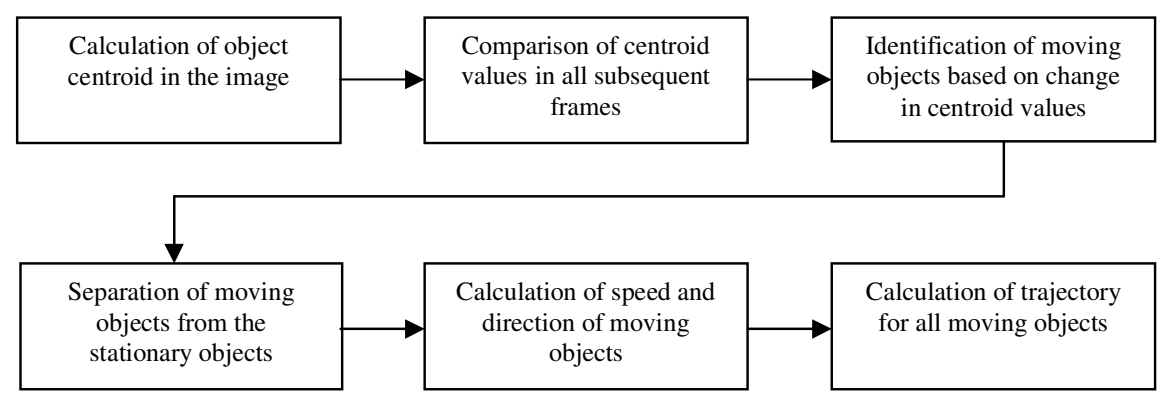

Fig.3 Block diagram of the complete model

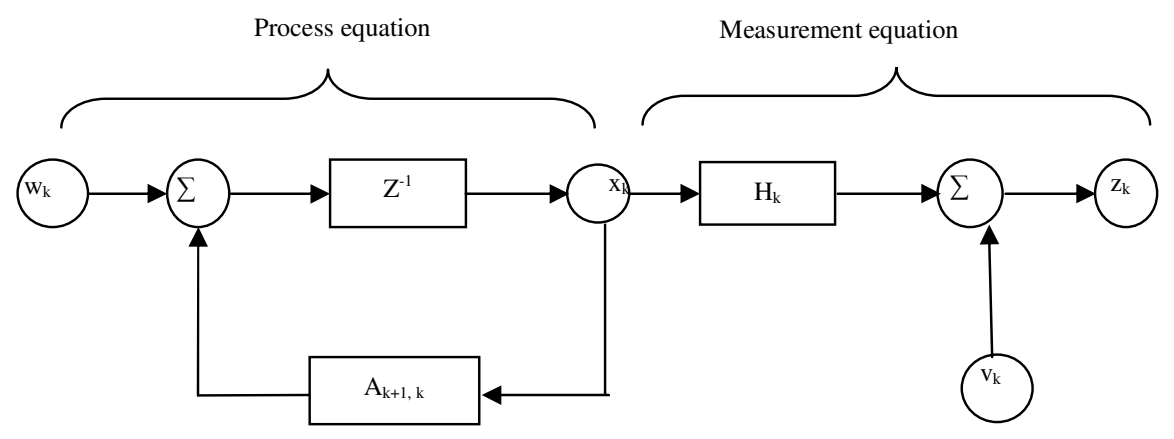

Fig. 4 Target aspects estimation and tracking from Kalman filter

transition, together with a non-linearmeasurement relationship. For the standard Kalman filter, the state transition from $k-1$ to $\mathrm{k}$ can be expressed with the equation

$$
x_{k}=A x_{k-1}+w_{k-1}
$$

where $\mathrm{A}$ is referred to as the state transition matrix and $\mathrm{w}_{\mathrm{k}-1}$ is a noise term. This noise term is a Gaussian random variable with zero mean and a covariance matrix $Q$, so its probability distribution is 


$$
p(w) \sim N(0, Q)
$$

The covariance matrix $\mathrm{Q}$ will be referred to as the process noise covariance matrix in the remainder of this report. It accounts for possible changes in the process between $k-1$ and $k$ that are not already accounted for in the state transition matrix. Another assumed property of $w_{k-1}$ is that it is independent of the state $x_{k-1}$.

It is also necessary to model the measurement process, or the relationship between the state and the measurement. In a general sense, it is not always possible to observe the process directly (i.e. all the state parameters are observable without error). Some of the parameters describing the state may not be observable at all, measurements might be scaled parameters, or possibly a combination of multiple parameters. Again, the assumption is made that the relationship is linear. So the measurement $z_{k}$ can be expressed in terms of the state $x_{k}[10]$ with

$$
z_{k}=H x_{k}+v_{k}
$$

where $\mathrm{H}$ is an $\mathrm{m} \times \mathrm{n}$ matrix which relates the state to the measurement. Much like $\mathrm{w}_{\mathrm{k}-1}$ for the process, $\mathrm{v}_{\mathrm{k}-1}$ is the noise of the measurement. It is also assumed to have a normal distribution expressed by

$$
p(v) \sim N(0, R)
$$

where $\mathrm{R}$ is the covariance matrix referred to as measurement noise covariance matrix.

In our analysis, the state $x_{k}$ contains the position $(x, y)$ of the object at the instant $k$ and also the speed of the object in both $\mathrm{x}(\dot{x})$ and $\mathrm{y}(\dot{y})$ directions [11]. The new position $\left(x_{k}, y_{k}\right)$ is the old position $\left(x_{k-1}, y_{k-1}\right)$ plus the velocity $\left(\dot{x}_{k-1}, \dot{y}_{k-1}\right)$ plus noise $w_{k-1}$.

The state equation, Eq. (5) is then in this case is defined as

$$
\left[\begin{array}{c}
x_{k} \\
y_{k} \\
\dot{x}_{k} \\
\dot{y}_{k}
\end{array}\right]=\left[\begin{array}{cccc}
1 & 0 & t & 0 \\
0 & 1 & 0 & t \\
0 & 0 & 1 & 0 \\
0 & 0 & 0 & 1
\end{array}\right]\left[\begin{array}{l}
x_{k-1} \\
y_{k-1} \\
\dot{x}_{k-1} \\
\dot{y}_{k-1}
\end{array}\right]+w_{k-1}
$$

Where ' $t$ ' represents the time interval between any two successive image frames and is considered as 1 second. And the measurement equation Eq. (6) is written as

$$
z_{k}=\left[\begin{array}{c}
x_{\text {kmeas }} \\
y_{\text {kmeas }}
\end{array}\right]=\left[\begin{array}{cccc}
1 & 0 & 0 & 0 \\
0 & 1 & 0 & 0
\end{array}\right]\left[\begin{array}{c}
x_{k} \\
y_{k} \\
\dot{x}_{k} \\
\dot{y}_{k}
\end{array}\right]+v_{k}
$$

Where $x_{\text {kmeas }}$ and $y_{\text {kmeas }}$ are the measured positions in $x$ and $y$ directions.

The Kalman filter estimates a process by using a form of feedback control: the filter estimates the process state at some time and then obtains feedback in the form of (noisy) measurements. As 
such, the equations for the Kalman filter fall into two groups: time update equations and measurement update equations. The time update equations are responsible for projecting forward (in time) the current state and error covariance estimates to obtain the priori estimates for the next time step. The measurement update equations are responsible for the feedback

i.e. for incorporating a new measurement into the priori estimate to obtain an improved posteriori estimate.
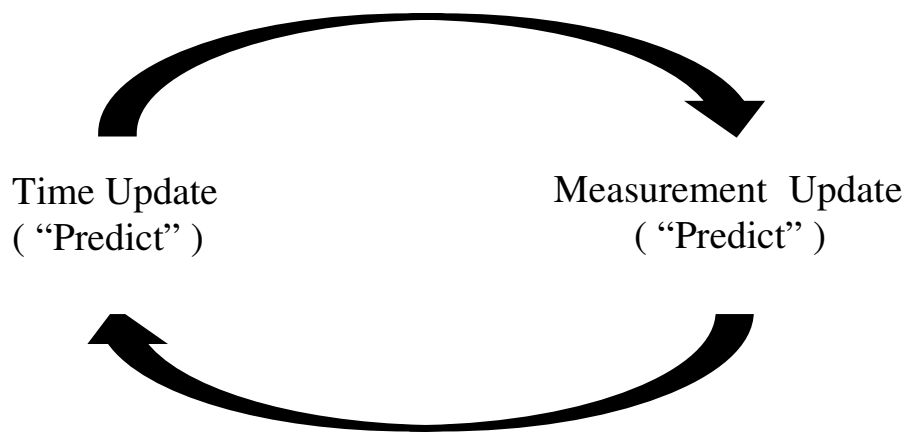

Figure 5. Kalman filter cycle

As shown in Figure 5, the time update projects the current state estimate ahead in time and the measurement update adjusts the projected estimate by an actual measurement at the time.

The time update equations can also be thought of as predictor equations, while the measurement update equations can be thought of as corrector equations.

The main steps of Kalman Filtering algorithm that has been implemented in this paper are as follows:-

Time Update ( Predict ) equations:

Step 1: Project the state ahead:

$$
\hat{x}_{k}^{-}=A \hat{x}_{k-1}+w_{k}
$$

Step 2: Project the error covariance ahead:

$$
P_{k}^{-}=A P_{k-1} A^{T}+Q
$$

Measurement Update ( Correct ) equations:

Step 3: Compute the Kalman gain:

$$
K_{k}=P_{k}^{-} H^{T}\left(H P_{k}^{-} H^{T}+R\right)^{-1}
$$

Step 4: Update estimation with measurements:

$$
\hat{x}_{k}=\hat{x}_{k}{ }^{-}+K_{k}\left(z_{k}-H \hat{x}_{k}{ }^{-}\right)
$$

Step 5: Update the error covariance:

$$
P_{k}=\left(I-K_{k} H\right) P_{k}^{-}
$$

Step 6: Go to Step 1. 
Steps 1 and 2 are responsible for projecting forward (in time) the current state and error covariance $\left(P_{k}^{-}\right)$estimates to obtain the a priori $\left(\hat{x}_{k}{ }^{-}\right)$estimates for the next time step. Steps 3 to 5 are responsible for the feedback i.e. for incorporating a new measurement into the a priori estimate to obtain an improved a posteriori estimate $\left(\hat{x}_{k}\right)$.The Kalman gain $K_{k}$ i.e. equation (9) in (step 3) is chosen to be the gain that minimizes the posteriori error covariance. The next step is to actually measure the process to obtain $z_{k}$, and then to generate a posteriori state estimate by incorporating the measurement as in equation (10). The final step is to obtain a posteriori error covariance estimate via equation (11).

After each time and measurement update pair, the process is repeated with the previous posteriori estimates used to project or predict the new priori estimates. This recursive nature is one of the very appealing features of the Kalman filter. It makes practical implementations much more feasible than (for example) an implementation of a Wiener filter (Brown and Hwang 1996) which is designed to operate on all of the data directly for each estimate. The Kalman filter instead recursively conditions the current estimate on all of the past measurements.

\section{DATA ASSOCiATION}

In many applications, knowledge of which target in the current frame relates to which target in the previous frame is important and so the data association problem would need to be addressed. Traditional multi-target tracking is based on coupling trackers such as Kalman filters, extended Kalman filters or particle filters with a data association technique (Bar-Shalom [12] provides a comprehensive treatment). The aim of the data association process is to interpret which measurements are due to the targets and which are due to false alarms. An example of this used on forward-looking sonar data is shown in [13]. Another technique which has been applied to sonar imagery uses Optical Flow calculations to estimate direction motion [14].

Data Association plays a very important role in all tracking applications, especially in the environment which is heterogeneous and having frequent occlusion conditions. This is aptly applicable to the undersea environment where AUV operates.

To check that the objects those have appeared in the subsequent images actually belong to the same target or not, following two conditions have been considered in our model [6]:-

a. The maximum pixels by which the centroid values will change in subsequent frames, if the objects are moving with the maximum speed.

b. The variation in the position of the centroid due to the movement of water body/ occlusion.

For calculating the maximum pixels by which the centroid values will change in subsequent frames, following model has been proposed:-

a. Assume, maximum speed that any object can have is ' $X$ ' $\mathrm{km} / \mathrm{hr}$.

b. The time interval between every two subsequent frames is ' $n$ ' seconds.

c. Image size is assumed to be kxk (for example 600x600).

d. The Range of the Sonar ( $R$ ) has been taken in terms of meters.

Based on the above inputs, the maximum amount of distance (in meters) that an object will cover when it is moving with maximum speed (X) is calculated. The distance is calculated as follows:- 
a. Maximum distance that will be covered by the objects between consecutive frames is $[n *(X * 1000 / 3600)]$ Meters

b. The resolution of the image is $\mathrm{R} / \mathrm{k}$ meters per pixels both in row and column.

Therefore the maximum distance that will be covered in terms of pixels is given by

$$
\left[\left(\mathrm{X}^{*} 1000 / 3600\right) * \mathrm{n}\right] *[\mathrm{R} / \mathrm{k}]
$$

In this paper, following values have been selected:-

Maximum speed of the object: $X=8 \mathrm{~km} / \mathrm{hr}$.

Time difference between two subsequent frames as 1 sec.

The Size of the Sonar image as 600x600.

The Sonar range $(\mathrm{R})$ as 10 meters.

The resolution of the image is therefore $(\mathrm{R} / \mathrm{k}) 10 / 600=0.0167$ meters/pixel for both row and column pixels.For any specified range up to $300 \mathrm{mts}$, sonar can scan 0 o to $360^{\circ}$. While taking the sonar data the sector is limited to $120^{\circ}$ and the range is kept to $10 \mathrm{mts}$. Since the image size is of $600 \times 600$ where each column containing 600 pixels exactly covers $10 \mathrm{mts}$ range. Hence in any direction throughout the $120^{\circ}$ sector of the image, the resolution of the image is $0.0167 \mathrm{mts} / \mathrm{pixel}$.

By substituting the above values in the Eq. (12), the maximum distance in terms of meters that will be covered by the object for every 1 second is calculated as 8.889 meters. Therefore, if the distance between the centroids of object in the consecutive images is less than or equal to the 8.889 meters then it is assumed to be from the same object i.e. they are said to be associated. Inherently, there is a variation in the position of the centroid due to the movement of water body. By comparing the centroid values of the associated object in subsequent frames, if the difference between the centroids is non-zero and is also greater than the variation in the position of the centroid due to the movement of water body, object is said to be associated and moving. Similarly if the centroid remains within the specified values (depends on the water body movement) in all subsequent frames then it is assumed to be stationary. The speed of the moving object is calculated accordingly.

Once the data association has been applied, we can initiate the tracking. Three cases are then possible:

a. There is a new observation matching the predicted position. The Kalman filter recursion is applied, a new state vector derived and new internal values computed.

b. No new observation matches the prediction. The obstacle prediction is updated using the Kalman filter internal values which are not updated. If no match is found between the observations and a given tracked object on a predefined number of frames, the tracked object is discarded as a false alarm.

c. An observation is not associated with any tracked object, a new object is created and its corresponding Kalman filter initialized.

By invoking the tracking algorithm for the moving objects, their trajectories are then calculated.

\section{Results And Data Processing}

To acquire the required data, several experiments have been conducted at the Towing Tank of the DRDO (NSTL), Visakhapatnam. The experiments included different scenarios such as object is moving and Sonar remains stationary; Sonar is moving and object remains stationary; and both Sonar and object are moving. The Towing tank is 500 meters in length with 8 meters in depth. In 
this paper, we have implemented the proposed algorithm for the specific case i.e. object moving and Sonar remains stationary. In this experiment, Digital Sector Scan (DSS) Sonar is fixed to carriage and kept stationary at one place in the water, and object is moved manually towards the Sonar. During data collection, the sector of Sonar is set to $120^{\circ}$. The recorded video was then converted into image frames using the 'SeanetImageOut.exe' software supplied along with the Sonar. For the real-time implementation of the proposed algorithm, the resolution is kept low so as to get the images instaneously at high speed.

Table 1. Comparison of Measured and Estimated Object positions (CENTROIDS)

\begin{tabular}{|l|ll|lc|}
\hline \multicolumn{3}{|c|}{ Measured positions } & \multicolumn{2}{c|}{ Estimated positions } \\
\hline Frames & \multicolumn{2}{|c|}{$\begin{array}{c}\mathbf{y} \\
\text { coordinates }\end{array}$} & \multicolumn{2}{c|}{$\mathbf{x}$ coordinates } \\
\hline Frame1 & 317.3450 & 107.5848 & 317.3561 & 107.5907 \\
\hline Frame2 & 368.2032 & 187.0239 & 368.1796 & 187.0015 \\
\hline Frame3 & 473.6369 & 251.8636 & 474.2643 & 252.2195 \\
\hline Frame4 & 475.9537 & 288.6233 & 475.6405 & 288.4390 \\
\hline Frame5 & 394.5096 & 336.3308 & 393.3391 & 335.6495 \\
\hline Frame6 & 359.0980 & 398.4558 & 358.3820 & 398.0353 \\
\hline Frame7 & 370.5565 & 463.4487 & 370.2715 & 463.2771 \\
\hline
\end{tabular}

The images were generated at every 1 second interval. These images were segmented so as to identify the object and this was followed by the estimation of trajectory using Kalman Filter. In the proposed model the objects have been associated by using the Data Association algorithm as discussed in section 2.2. From the results the Mean Square Error for x-coordinate is found to be 0.3509 meters and for the y-coordinate is found to be 0.1188 meters. The accuracy of the object positions is found to be \pm 0.0486 meters. Table 1 shows the measured and estimated positions (in terms of pixel values) of the objects in 7 subsequent frames. The final result has been shown in Figure 5 which is a polar plot shows tracking of the object using the measured as well as the estimated positions. From the proposed model, it is observed that time taken to identify the objects through segmentation and extraction of obstacle parameters such as their range, bearing, size, shape, speed and course using Kalman filter is approximately 0.4 seconds which is reasonably less and aptly applicable for the application of obstacle avoidance. For every frame, the complete processing takes 0.4 seconds and the time interval between the input frames is 1 second.

The sonar used to collect the data is Super Seeking DST (Digital Sonar Technology) Dual Frequency CHIRP Sonar. Sector scan sonar with the following specifications:

Operating frequency (low)-Chirping from 250 to $350 \mathrm{kHz}$ (300)

Operating frequency (high)-Chirping from 620 to $720 \mathrm{kHz}$ (670)

Optional high frequency $\quad 1 \mathrm{MHz}$

Beamwidth, vertical $20^{\circ}[300]$

Beamwidth, vertical $\quad 40^{\circ}[670]$

Beamwidth, horizontal $\quad 3.0^{\circ}[300]$ 


$\begin{array}{ll}\text { Beamwidth, horizontal } & 1.5^{\circ}[670] \\ \text { Maximum range } & 300 \mathrm{~m}[300] \\ \text { Maximum range } & 100 \mathrm{~m} \mathrm{[670]} \\ \text { Minimum range } & 0.4 \mathrm{~m} \\ \text { Scanned sector } & \text { Variable to } 360^{\circ} \\ \text { Range resolution } & 5-400 \mathrm{~mm} \text { depending on range }\end{array}$

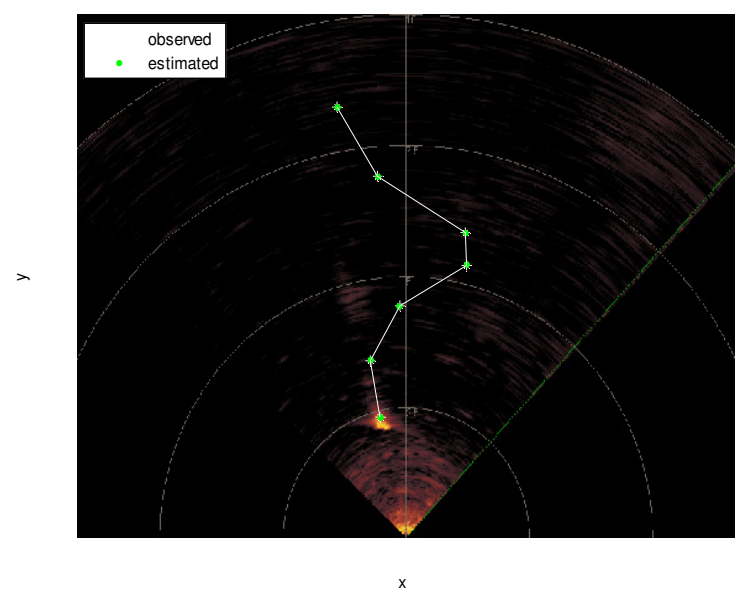

Figure 6. Object Tracking Using Kalman

\section{CONCLUSIONS}

Considering the fact that the time taken for calculation of target parameters for the purpose of obstacle avoidance must be as less as possible and the position should be as accurate as possible, the algorithm that has been proposed and validated in this paper can be concluded to meet the given requirement. It is seen from the results that the time taken for undertaking complete processing on every image is approximately 0.4 Seconds and the positional accuracy is found to be \pm 0.0486 meters. It implies that if the obstacle is detected at the range of 300 meters and if the AUV is moving with the speed of $8 \mathrm{Km} / \mathrm{Hr}$ then the sufficient time is available with the AUV to take corrective course of action which is approximately 150 Seconds if the object is stationary and approximately 75 Seconds if the object is moving head on with the same speed as AUV. Therefore it can be concluded that the algorithm proposed in this paper is aptly suited for the application of obstacle Avoidance in case of AUV navigating in constrained underwater scenario.

\section{ACKNOWLEDGEMENTS}

The above work has been undertaken towards the research project of DRDO (NSTL). The authors are thankful to the project team at NSTL for providing the SONAR data, constant technical support and encouragement. Authors are also thankful to the management of their respective organizations. 
International Journal of Computer Science, Engineering and Information Technology (IJCSEIT), Vol.2, No.2, April 2012

\section{REFERENCES}

[1] Michael. J. Smith "Bayesian Sensor Fusion a framework for using multi-modal sensors to estimate target location and identities in a battlefield scene" PhD thesis, Florida state University, 2003.

[2] Bir Bhanu, "Automatic Target Recognition: State of the Art Survey", IEEE transactions on aerospace and electronics systems, vol. AES-22, No.4 july 1996.

[3] Yifeng Zhu and Ali Shareef, "Comparisons of Three Kalman Filter Tracking Algorithms in Sensor Network", this work was supported by National Science Foundation grant 0538457 and an UMaine Startup Fund.

[4] I.T. Ruiz, Y. Petillot, D. Lane, J. Bell, "Tracking objects in underwater multibeam sonar images," IEE Colloquium on Motion Analysis and Tracking, London, UK, 10th May 1999, pp.11/1-11/7.

[5] I.Tena Ruiz, Y. Petillot, D. M. Lane, C. Salson "Feature Extraction and Data Association for AUV Concurrent Mapping and Localisation" Proceedings of the 2001 IEEE International Conference on Robotics \& Automation Seoul, Korea, May 21-26, 2001.

[6] Xu, L.; Landabaso, J. L.; Lei, B.; "Segmentation and tracking of multiple moving objects for intelligent video analysis", BT Technology Journal, Vol 22, No 3, July 2004.

[7] S. Blackman and R. Popoli, Design and Anal- ysis of Modern Tracking Systems, Boston, MA, Artech, pp. 157-160, 1999.

[8] A. S. Gelb, Applied Optimal Estimation, Cambridge, MA, MIT Press, 1974.

[9] Y. Bar-Shalom and T. E. Fortmann, Tracking and Data Association, Orlando, FL, Academic Press, 1988.

[10] T.H. Lee, W.-S. Ra, T.S. Yoon and J.B. Park, "Robust Kalman filtering via Krein space estimation”, IEE Proc.-Control Theory Appl., Vol. 151, No. 1, January 2004.

[11 Yvan Petillot, Ioseba Tena Ruiz, and David M. Lane, "Underwater Vehicle Obstacle Avoidance And Path Planning Using a Multi-Beam Forward Looking Sonar,” IEEE journal of Oceanic Engineering, vol.26, No.2, April 2001.

[12] Y. Bar-Shalom and T.E. Fortmann. Tracking and Data Association. Academic Press, 1988.

[13] E. Trucco, Y. Petillot, I. Tena Ruiz, C. Plakas, and D. M. Lane. Feature tracking in video and sonar subsea sequences with applications. Computer Vision and Image Understanding, No.79, pages 92.122.16

[14] I. Tena Ruiz, D. M. Lane, and M. J. Chantler. A comparison of inter-frame feature measures for robust object classi_cation in sector scan sonar image sequences. IEEE Journal of Oceanic Engineering, 24, No.4:458.469, 1999.

[15] Cushieri J. and Negahdaripour S., "Use of forward scan sonar images for positioning and navigation by an AUV”, in Proceedings of OCEANS'98, Nice, France, (IEEE/OES, Ed.), Vol. 2, pp:752-756, September 1998.

[16] Bell J.M., Dura E., Reed S., Petillot Y.R., and Lane D.M., "Extraction and Classification of Objects from Sidescan Sonar", IEE Workshop on Nonlinear and Non-Gaussian Signal Processing, 8-9th July 2002.

[17] Clark D., Ruiz 1.T., Petillot Y. and Bell J., "Multiple Target Tracking and Data Association in Sonar Images", The IEE Seminar on Target Tracking: Algorithms and Applications 2006 (Ref. No. 2006/11359), Birmingham, UK, pp: 147- 154, March 2006.

[18] Clark D.E. and Bell J., "Bayesian multiple target tracking in forward scan sonar images using the PHD filter", Radar, Sonar and Navigation, IEE Proceedings, Vol.152, Issue 5, pp:327 - 334, October 2005. 


\section{Authors}

Smt Nagamani Modalavalasa received B.E. degree in Electronics and Communication Engineering from Andhra University, Visakhapatnam, Andhra Pradesh, India. She has completed her Master degree from Andhra University, Visakhapatnam, India. At present she is a research scholar in Electronics \& Communication Engg. Department, JNTU Engg. College, Kakinada, Andhra Pradesh, India. She has 15 years of teaching experience as Lecturer in the Department of Electronics \& Communication Engg, State Board of Technical Education \& Training, Andhra Pradesh, India. She has published around 15 research papers in various International and National conferences.

Dr G Sasi Bhushana Rao received B E. degree in Electronics and Communication Engineering from Andhra University College of Engineering, Visakhapatnam, Andhra Pradesh, India, M.Tech. degree from JNTU, Hyderabad, India and Ph.D. from Osmania University, Hyderabad, India. He possesses vast administration, teaching and R\&D experience at Airports Authority, Andhra University, India for about 25 years. Currently he is working as Head Of Department in the Department of Electronics \& Communication Engg, Andhra University Engineering College, Visakhapatnam, India. He has published more than 230 Technical and research papers in different National / International conferences and Journals and authored two Text books. He has guided 4 Ph.D. scholars and at present 14 scholars are working with him. His areas of Research include Inertial Navigation System (INS), GPS/GNSS Signal processing, Ionosphere/Troposphere and Multipath error modeling, and RADAR and SONAR navigation. Dr. Rao is a Fellow member of various professional bodies like IEEE, IETE, IGU, and International GNSS society.

Dr. K. Satya Prasad received B Tech. degree in Electronics and Communication Engineering from JNTU college of Engineering, Anantapur, Andhra Pradesh, India, M.E. degree in Communication Systems from Guindy college of Engg. , Madras University, Chennai, India and $\mathrm{Ph}$. D from Indian Institute of Technology, Madras. $\mathrm{He}$ has more than 31 years of experience in teaching and 23 years of $\mathrm{R} \& \mathrm{D}$. He started his teaching carrier as Teaching Assistant at Regional Engineering College, Warangal in 1979. He joined JNT University, Hyderabad as Lecturer in 1980 and served in different constituent college's viz., Kakinada, Hyderabad and Anantapur and at different capacities viz., Associate Professor, Professor, and Head of the

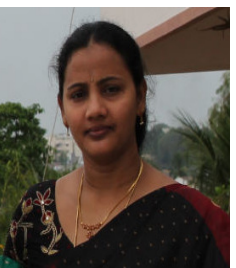
Department, Vice Principal and Principal. He has published more than 50 technical papers in different National / International conferences and Journals and authored one Text book. He has guided 4 Ph.D. scholars and at present 12 scholars are working with him. His areas of Research include Communications Signal Processing, Image Processing, Speech Processing, Neural Networks \& Ad-hoc wireless networks etc. Dr. Prasad is a Fellow member of various professional bodies like IETE, IE, and ISTE. 